\title{
microRNA-103 regulates the growth and invasion of endometrial cancer cells through the downregulation of tissue inhibitor of metalloproteinase 3
}

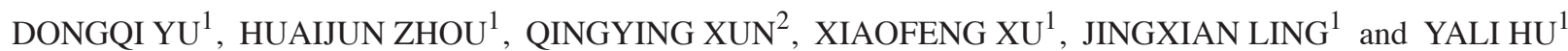 \\ ${ }^{1}$ Department of Gynecology and Obstetrics, The Affiliated Drum Tower Hospital of Nanjing University Medical School; \\ ${ }^{2}$ Department of Physiology, Medical College of Southeast University, Nanjing, Jiangsu, P.R. China
}

Received November 29, 2011; Accepted February 21, 2012

DOI: $10.3892 / \mathrm{ol} .2012 .638$

\begin{abstract}
Despite improvements in treatment over the past few decades, endometrial cancer remains one of the most common causes of mortality in women and there is an urgent need for the development of targeted therapies. The aim of this study was to confirm the target gene of miR-103 in human endometrial cancer and investigate the biological functions in which miR-103 is involved through the regulation of the expression of its target gene. This study may provide useful data to gain a better understanding of the effect of miR-103 in tumor formation. miR-103 expression levels were measured using real-time quantitative PCR. The effect of miR-103 on tissue inhibitor of metalloproteinase 3 (TIMP-3) expression was assessed in endometrial cancer cell lines with a miR-103 inhibitor to decrease the level of miR-103 expression. Furthermore, the roles of miR-103 in cell growth and invasion were analyzed using miR-103 inhibitor-transfected cells. The level of expression of miR-103 decreased following transfection with the miR-103 inhibitor. miR-103 inhibitor transfection increased the activity of the luciferase reporter assay containing the TIMP-3 3'-untranslated region (UTR) construct and increased the levels of the TIMP-3 protein but not its mRNA in endometrial cancer cell lines. Finally, miR-103 inhibitor-transfected cells exhibited reduced cell growth and invasive characteristics. Our data suggested that miR-103 post-transcriptionally downregulates the expression of the tumor suppressor TIMP-3 and stimulates growth and invasion in endometrial cancer cell lines. This provides a possible therapeutic target that may upregulate TIMP-3 in endometrial cancer.
\end{abstract}

Correspondence to: Dr Huaijun Zhou, Division of Gynecologic Oncology, Department of Gynecology and Obstetrics, The Affiliated Drum Tower Hospital of Nanjing University Medical School, Nanjing, Jiangsu 210008, P.R. China

E-mail: zhouhj2007@126.com

Key words: miR-103, endometrial cancer, tissue inhibitor of metalloproteinase 3, Ishikawa cells, HEC-1B cells

\section{Introduction}

Endometrial cancer is one of the most common types of female cancer and it seriously endangers the health of women. Therefore, research on the pathogenesis and novel therapeutic approaches to endometrial cancer is of great significance. microRNAs (miRNAs) are a class of small non-coding RNAs of approximately 22 nucleotides in length, which regulate gene expression in animals and plants by targeting mRNAs at the 3'-untranslated regions (UTRs) for cleavage or translational repression. The results of previous studies showed that over half of the miRNAs may be aligned to genomic fragile sites or regions associated with cancer (1), indicating that miRNA may be significant in cancer pathogenesis, development and metastasis. The aberrant expression of miRNA has been reported in various tumors, including lung, colon, breast, liver and gastric cancer (2-5), indicating that there is a close correlation between miRNAs and human malignancy.

The aberrant overexpression of miR-103 was first identified in endometrial cancer by Boren et al (6) and Chung et al (7). Boren et al found that miR-103 may be associated with the development of endometrial cancer and suggested tissue inhibitor of metalloproteinase 3 (TIMP-3) as a potential target for miR-103. Later studies suggested that miR-103 was aberrantly overexpressed in various types of cancer. For example, 10 miRNAs, including miR-103, were found to be upregulated in bladder cancer (8). miR-103 maps near the homeobox (HOX) genes, which are crucial for normal development and oncogenesis. Yang et al (9) reported that the overexpression of miR-103 may be involved in the tumorigenesis of erythroleukemia by arresting erythroid maturation. Guo et al (10) found that the high expression of has-miR-103/107 was correlated with poor survival by univariate and multivariate analyses. More recently, the results of other studies have indicated that miR-103 affects neuronal migration by modulating CDK5R1 expression (11). However, as yet, no studies have investigated the effects of miR-103 in endometrial cancers. The mechanism by which miR-103 affects cancer development and progression is not fully understood. Previous studies suggested that the attenuated expression of TIMP-3 exists in various types of cancer, including chorionic, prostatic and esophageal carcinomas and melanotic cancer (12-15). At present, no studies 
investigating TIMP-3 expression in endometrial cancer have been published.

In this study, we aimed to evaluate the possible involvement of miR-103 in endometrial carcinogenesis and to reveal any correlation between miR-103 and TIMP-3. The results of this study may aid the understanding of the effects of miR-103 in the tumorigenesis and progression of endometrial cancer.

\section{Materials and methods}

Cell culture and reagents. The human endometrial cancer cell lines, HEC-1B (estrogen receptor-negative; from our laboratory) and Ishikawa (estrongen receptor-positive; donated by Professor Wei Lihui, Medical College of Beijing University) were grown in $\alpha$-MEM (Gibco, Darmstadt, Germany) supplemented with $10 \%$ fetal bovine serum (Gibco), $100 \mathrm{U} / \mathrm{ml}$ of penicillin and $100 \mu \mathrm{g} / \mathrm{ml}$ of streptomycin (Gibco). The cells were incubated at $37^{\circ} \mathrm{C}$ in a humidified chamber supplemented with $5 \% \mathrm{CO}_{2}$. The anti-miR-103 oligonucleotide and control negative oligonucleotide (negative control) were purchased from Ambion (Carlsbad, CA, USA). The TIMP-3 antibody was purchased from Abcam (Cambridge, MA, USA), GAPDH antibody from Sigma (Steinheim, Germany) and goat anti-mouse IgG from Bio-Rad (Hercules, CA, USA). The primer sequences used were: miR-103 RT primer: GTCGTATCCAGTGCAGGGTCCGAGGTAT TCGCACTGGATACGACTCAGCC; miR-103 forward: GAGCAGCATTGTACAG and reverse: GTGCAGG GTCCGAGGT: U6 forward: CTCGCTTCGGCAGCACA and reverse: AACGCTTCACGAATTTGCGT; TIMP-3 forward: AGTTACCCAGCCCTATGA and reverse: GCA AAGGCTTAAACATCT; $\beta$-actin forward: CGTGGG CCGCCCTAGGCACCA and reverse: TTGGCTTAGGG TTCAGGGGGG. The primers were purchased from Shanghai Songon (Shanghai, China). Transwell chambers $(8 \mu \mathrm{m}$, $6.5 \mathrm{~mm}$ ) were purchased from Millipore (Billerica, MA, USA) and Matrigel was purchased from BD Biosciences (San Jose, CA, USA).

Cell transfection. Suitable cells were seeded in six-well plates to ensure that they would reach $30 \%$ confluence the following day. The transfection of the anti-miR-103 or negative control oligonucleotide was performed using the Lipofectamine 2000 reagent (Invitrogen, Carlsbad, CA, USA) in antibiotic-free Opti-MEM (Invitrogen) according to the manufacturer's instructions, with a final concentration of $15 \mathrm{nM}$ in the Ishikawa cells and $30 \mathrm{nM}$ in the HEC-1B cells. Following $48 \mathrm{~h}$ of transfection, the cells were harvested and processed for further analysis.

Quantitative reverse transcription ( $q R T)-P C R$ analysis of $m R N A$ and miRNA expression. Total RNA was isolated from the cells using TRIzol reagent (Invitrogen) to obtain miRNA and mRNA. For the expression analysis of miR-103 and TIMP-3, the stem-loop RT and real-time PCR primers were designed as described above. Briefly, miRNAs and mRNA were examined by SYBR real-time qRT-PCR (Takara, Dalian, China) on a 7500 Real-Time PCR system (Applied Biosystems, Carlsbad, CA, USA) according to the manufacturer's instructions. The relative expression was calculated using the $\Delta \mathrm{CT}$ method and the expression of miR-103 was normalized using the $2^{-\Delta \Delta \mathrm{CT}}$ method (Pfaffl, 2001) relative to U6-snRNA and TIMP-3-mRNA was normalized to $\beta$-actin. The qRT-PCR assays were performed in duplicate and the data were presented as the mean \pm standard error of the mean (SEM).

Western blot analysis. Cell lysates were prepared in lysis buffer containing a protease inhibitor cocktail (Roche Diagnostics, Mannheim, Germany) following a 48-h transfection with either anti-miR-103 or the negative control oligonucleotide. The protein concentrations of the total cell lysates were measured using the Micro BCA protein assay kit (Pierce Biotechnology Inc., Rockford, IL, USA) and $80 \mu \mathrm{g}$ of total cell lysate per lane was separated by $10 \%$ SDS-PAGE, then transferred to a PVDF membrane (Millipore). The membrane was then soaked in blocking solution (1X TBS, $10 \%$ non-fat dried milk) for $2 \mathrm{~h}$ and incubated with anti-actin monoclonal antibody at $4{ }^{\circ} \mathrm{C}$ overnight. The following day, the membrane was washed with TBST six times for $1 \mathrm{~h}$, incubated with the secondary antibody (goat anti-mouse IgG; 1:5,000; Santa Cruz Biotechnology, Inc., Santa Cruz, CA, USA) for $2 \mathrm{~h}$ and washed with TBST six times for $1 \mathrm{~h}$. Finally, the enhanced chemiluminescence (ECL) system western blotting detection reagents (Millipore) were used according to the manufacturer's instructions and exposed to X-ray film.

Luciferase reporter assay. The 3'-UTR segments of TIMP-3 mRNA containing the miR-103 binding sites were amplified by PCR from human genomic DNA and inserted into the $\mathrm{Xbal}$ site of the pGL3 vector (Promega Corporation, Madison, WI, USA), designated pGL3-TIMP-3-wt. pGL3-PTEN-mut, which had mutations in the predicted miR-103 binding sites, was constructed as a template. The wild-type or mutant reporter (300 ng) was co-transfected into Ishikawa and HEC1B cells in 12-well plates with a Renilla plasmid (10 ng) using Lipofectamine 2000 (Invitrogen). At $6 \mathrm{~h}$ after transfection, the cells were transfected with anti-miR-103 or the negative control oligonucleotide. The reporter assay was performed $42 \mathrm{~h}$ post-transfection using the Dual luciferase assay system (Promega) and the firefly luciferase activity of each sample was normalized to the Renilla luciferase activity.

Cell invasion assays. Transwell invasion experiments were performed in 24-well Matrigel-coated invasion chambers. Approximately $2 \times 10^{4}$ cells in $0.2 \mathrm{ml}$ serum-free $\alpha$-MEM were added into each filter of the upper chamber and $0.6 \mathrm{ml}$ of complete $\alpha$-MEM was added to the lower chambers. Following culture for $24 \mathrm{~h}$, the cells on the upper side of the filters were removed with cotton-tipped swabs and the cells that had invaded into the lower side of the filter were fixed in methanol for $15 \mathrm{~min}$, stained with $0.05 \%$ crystal violet in PBS for $15 \mathrm{~min}$ and microscopically observed and counted in 5 fields of view at a magnification of $x 200$. The invasive activity of the cancer cells was expressed as the mean number of cells that invaded to the lower side of the filter and the results were shown as the mean \pm SD of the number of cells per field of view. The experiments were repeated at least three times.

Cell proliferation assays. Cell proliferation was assessed using an MTT assay. Cells $\left(5.0 \times 10^{3}\right.$ cells/well) were seeded 
A

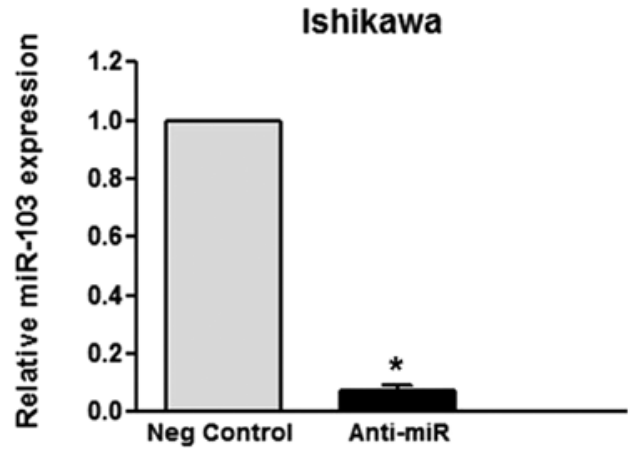

B

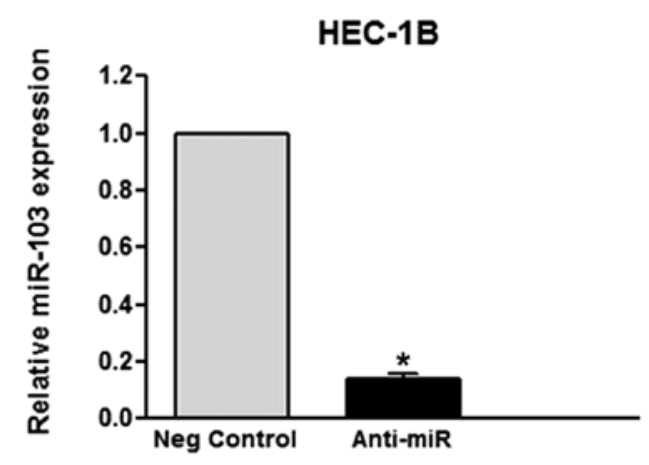

C

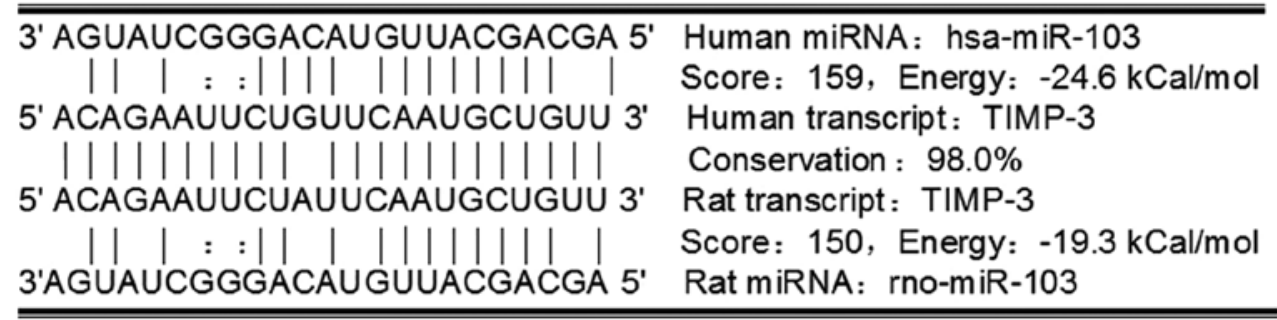

D

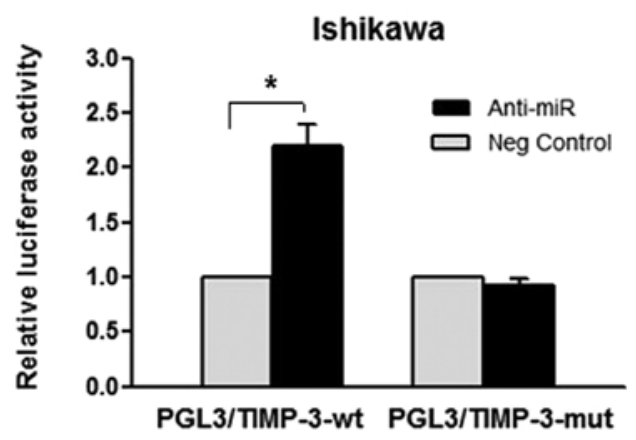

E

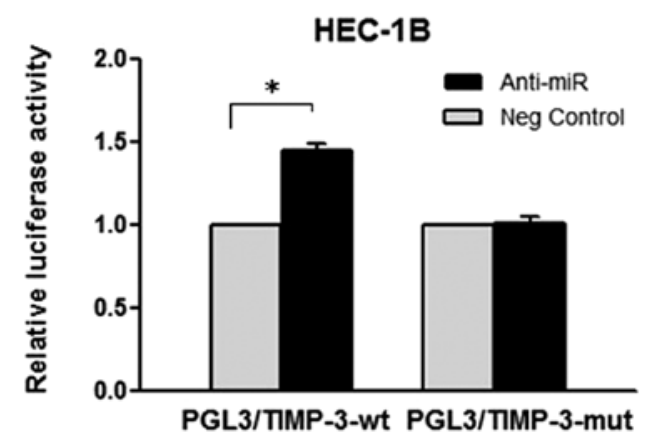

Figure 1. TIMP-3 3'-UTR is a direct target for miR-103. (A) Suppression of miR-103 by anti-miR-103 in Ishikawa cells is shown. The cells were frst transfected with anti-miR-103 or negative control oligonucleotide. The cells were harvested 2 days later and total RNA was extracted using TRIzol reagent. Mature miR103 was detected by SYBR qRT-PCR, using U6 RNA for normalization. (B) Suppression of miR-103 by anti-miR-103 in HEC-1B cells is shown. Following transfection with anti-miR-103 (Anti-miR) or negative control oligonucleotide (Neg Control), mature miR-103 was detected as in (A). (C) An evolutionarily conserved target sequence for miR-103 is detected in the 3'-UTR of TIMP-3. (D and E) The luciferase assay was performed using Ishikawa and HEC-1B cells, respectively. The effect of miR-103 on the luciferase activity of pGL3-TIMP-3-wt and pGL3-TIMP-3-mut was measured as described in Materials and methods. A statistically significant upregulation in luciferase activity was found when the cells were transfected with pGL3-TIMP-3-wt together with antimiR-103. pGL3-TIMP-3-wt, vector with wild-type TIMP-3 3'-UTR; pGL3-TIMP-3-mut, vector with mutated TIMP-3 3'-UTR. Each bar is the mean \pm SEM from three independent experiments. "P<0.05. qRT-PCR, quantitative reverse transcription PCR; UTR, untranslated region.

on 96-well plates. Following the transfection of the Ishikawa cells by anti-miR-103 for varying time periods $(20,44,68$ and $92 \mathrm{~h}$ ), $50 \mu \mathrm{l}$ of MTT ( $5 \mathrm{mg} / \mathrm{ml}$; Sigma, Chicago, IL, USA) was added to each well and the cells were incubated for a further $4 \mathrm{~h}$ at $37^{\circ} \mathrm{C}$. The plates were then agitated for $30 \mathrm{sec}$ and the optical density (OD) was measured at $490 \mathrm{~nm}$ on an enzyme immunoassay analyzer (Bio-Rad, Tokyo, Japan).

Evaluation of apoptosis. Apoptosis was detected by a flow cytometric analysis of Annexin V and PE staining. The Annexin V-FITC versus PE assay was performed following the manufacturer's instructions. Briefly, adherent cells were harvested and suspended in the binding buffer $\left(1 \times 10^{6}\right.$ cells $\left./ \mathrm{ml}\right)$. The cells were then incubated with Annexin V-FITC and PE for $15 \mathrm{~min}$ at room temperature in the dark and immediately analyzed by flow cytometry (BD Biosciences).

Statistical analysis. Statistical analysis was performed using the SPSS 13.0 software (SPSS, Inc., Chicago, IL, USA). The values are presented as the mean \pm SEM. The differences/ correlations between the groups were calculated using the Student's t-test and analysis of variance. $\mathrm{P}<0.05$ was considered to indicate a statistically significant result.

\section{Results}

miR-103 directlytargets TIMP-3 3'-UTRs. The mature sequence of miR-103 (5'-AGCAGCAUUGUACAGGGCUAUGA-3') was retrieved from the miRBase Sequence Database, release 14 (http://microrna.sanger.ac.uk/sequences/). A bioinformatics search (http://diana.pcbi.upenn.edu/cgi-bin/TargetCombo.cgi), revealed that an evolutionarily conserved target sequence for miR-103 is located in the 3'-UTR of TIMP-3 (Fig. 1C). This suggests that it is a target for miR-103. To validate whether TIMP-3 is a direct target of miR-103, we performed a luciferase reporter assay. The decreased level of expression of miR-103 upon transfection, confirmed by qRT-PCR (Fig. 1A and B), significantly affected the luciferase expression, measured as 
A

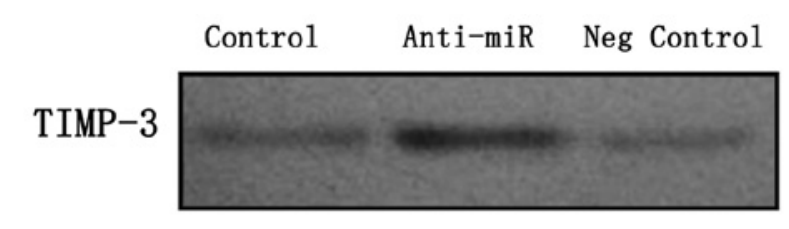

GAPDH

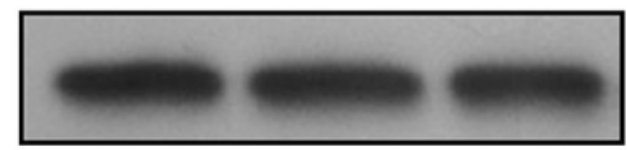

C

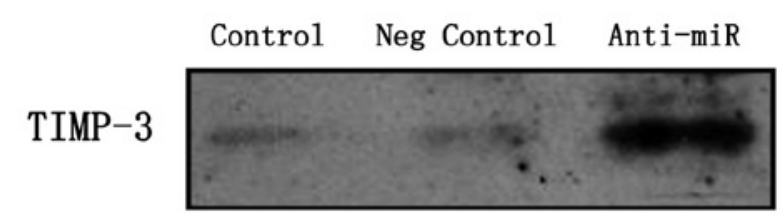

GAPDH

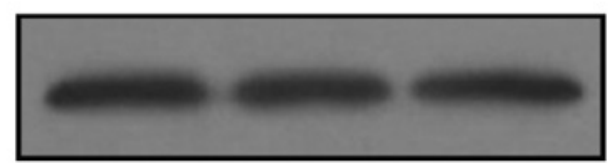

B

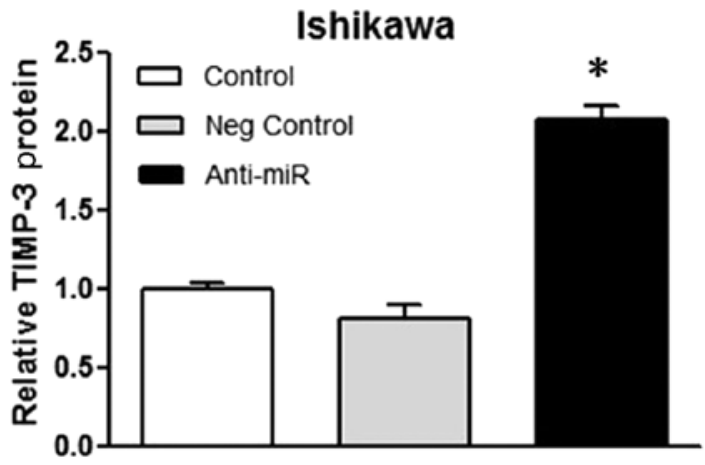

D

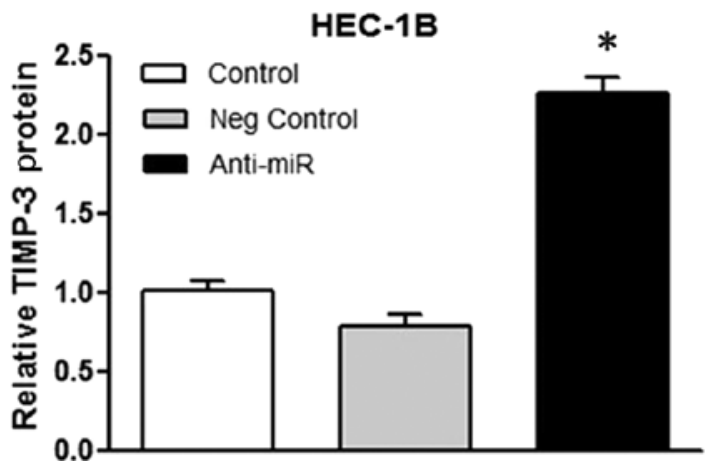

Figure 2. Inhibition of miR-103 upregulates TIMP-3 protein expression in endometrial cancer cells. Ishikawa and HEC-1B cells were transfected with anti-miR-103 (Anti-miR) or negative control oligonucleotide (Neg Control) and the TIMP-3 protein level was detected by western blot analysis. The GAPDH protein was regarded as an endogenous normalizer. (A and C) Inhibition of miR-103 upregulates the endogenous TIMP-3 protein expression in Ishikawa and HEC-1B cells. (B and D) A significant increase in TIMP-3 activity was detected in Ishikawa and HEC-1B cells transfected with anti-miR-103, compared with the other two groups. Each bar is the mean \pm SEM from three independent experiments. ${ }^{*} \mathrm{P}<0.05$.

relative luciferase activity (Fig. 1D and E). The results revealed a statistically significant upregulation of luciferase activity in Ishikawa and HEC-1B cells when these cells were transfected with pGL3-TIMP-3-wt together with the miR-103 inhibitor, but not with any other combination. These results confirm the bioinformatic predictions, indicating that the TIMP-3 3'-UTR is a direct target of miR-103.

Inhibition of miR-103 upregulates TIMP-3 protein expression in endometrial cancer cells. If miR-103 targets the TIMP-3 3'-UTR, the TIMP-3 protein expression levels should be inversely correlated with miR-103 expression levels. A western blot analysis of TIMP-3 expression was performed in two endometrial cancer cell lines, in which the knockdown of miR-103 expression following transfection was confirmed by qRT-PCR. As expected, the TIMP-3 protein levels were significantly increased in the two cell lines which were transfected with anti-miR-103, compared with those transfected with the negative control oligonucleotide and the untreated endometrial cancer cells (Fig. 2). These results are consistent with the hypothesis that miR-103 is able to target TIMP-3 and thereby influence the amount of TIMP-3 protein in the cells. We also measured the TIMP-3 mRNA expression by qRT-PCR, but the difference was not statistically significant (data not shown).

Inhibition of miR-103 reduces the growth and invasion of endometrial cancer cells. The impact of the modulation of miR-103 expression on cell growth and viability following transfection was tested using the MTT assay. No significant difference was observed in the light absorption values between the untreated and negative control groups (Fig. 3A). The cells that were transfected with anti-miR-103 showed slow proliferation and the inhibition rates at 48, 72 and $96 \mathrm{~h}$ following transfection were $20.70,40.63$ and $35.92 \%$, respectively, indicating that cell proliferation was suppressed by the transfection of anti-miR-103 (Fig. 3A). To evaluate the effect of miR-103 downregulation on the invasion of endometrial cancer cells, a cell invasion assay was performed. Cells were grown in the upper chamber and assessed for invasion through the Matrigel toward a chemoattractant (10\% serum) in the lower chamber. The number of invasive HEC-1B and Ishikawa cells transfected with anti-miR-103 were significantly reduced relative to those with control oligonucleotide (Fig. 3A and B). miR-103 silencing resulted in a $42.86 \%$ decrease in the number of invasive Ishikawa cells (Fig. 3C; $\mathrm{P}<0.05$ ). Similarly, the silencing of miR-103 in HEC-1B cells significantly inhibited cell invasion, with a $45.45 \%$ decrease in the number of invasive cells (Fig. 3C; $\mathrm{P}<0.05$ ). The Annexin V-FITC versus PE assay showed that anti-miR-103 had no effect on the apoptosis of the Ishikawa and HEC-1B cells. Taken together, these data suggest that miR-103 is essential for tumor cell growth and invasion in vitro.

\section{Discussion}

This is the first study to show that the tumor suppressor TIMP-3 is negatively regulated by miR-103 at the post-transcriptional 
A

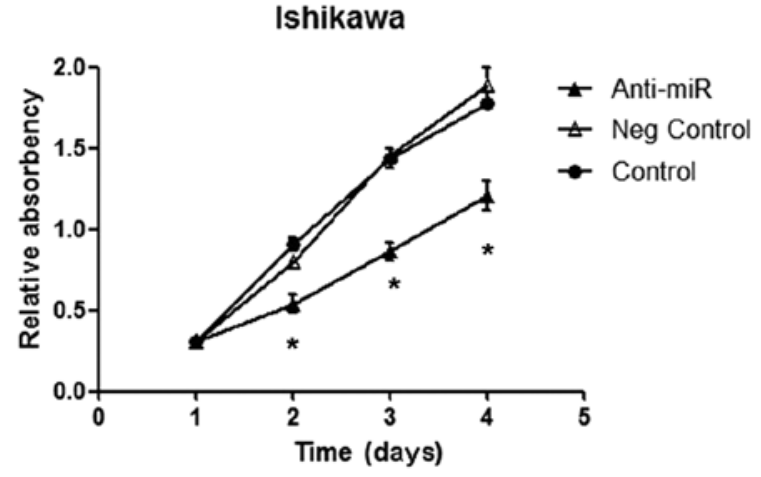

B

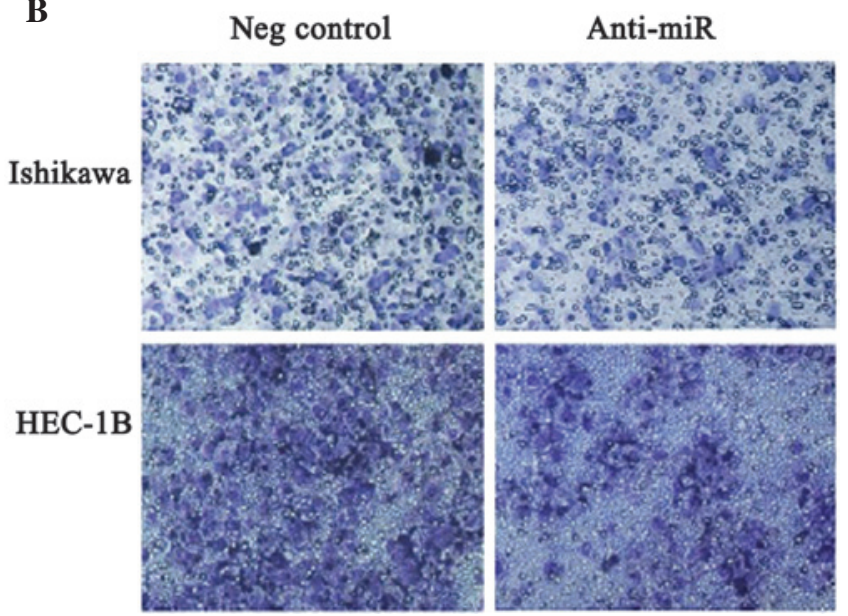

C

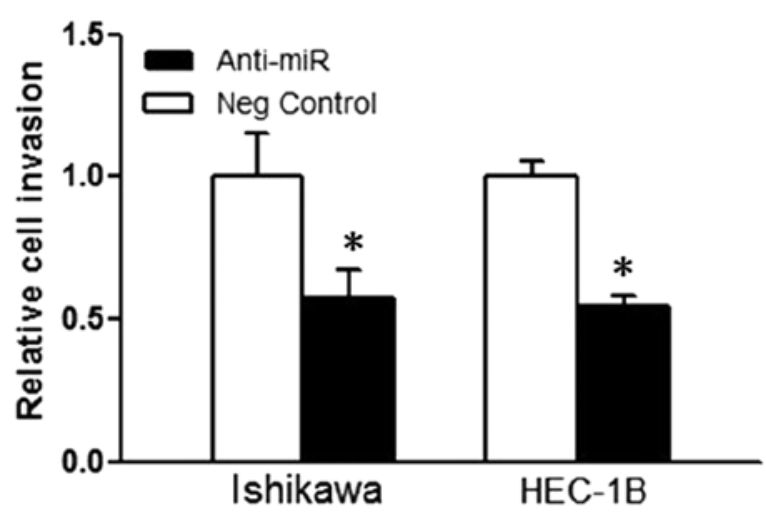

Figure 3. miR-103 affects endometrial cancer cell growth and invasion in vitro. (A) MTT assay was performed. The cells in the anti-miR-103 group showed slow proliferation compared with the untreated and negative control groups. The Ishikawa and HEC-1B cells were first transfected with antimiR-103 (Anti-miR) or negative control oligonucleotide (Neg Control) and then subjected to Matrigel chamber assays, as described in Materials and methods. After $48 \mathrm{~h}$, the invasive cells were counted following staining with crystal violet. (B) Representative fields of invasive cells on the membrane. (C) The data are standardized against the negative control and presented as relative cell invasion numbers. Each bar is the mean \pm SEM from three independent experiments. ${ }^{*} \mathrm{P}<0.05$.

level, via a specific target site within the 3 '-UTR. This is also the first study to demonstrate that miR-103 induces growth and invasion in endometrial cancer cells.

miRNAs induce the degradation or translational repression of target mRNAs and are thereby involved in the initiation and progression of human cancer (16), although the underlying mechanism is largely unknown.

To investigate the correlation between TIMP-3 and miR-103, we first performed a bioinformatics search. It revealed a conserved target site for miR-103 within the TIMP-3 3'-UTR. A miR-103 inhibitor was then transfected into the endometrial cancer cells. As expected, the epigenetic inhibition of the expression of miR-103 resulted in the upregulation of the TIMP-3 protein. To analyse whether the predicted miR-103 target site in the 3'-UTR of TIMP-3 was responsible for its regulation, we performed a luciferase reporter assay, the results of which were in accordance with those of the western blot analysis. These data suggest that miR-103 is important in the regulation of TIMP-3. Additionally, the aberrant expression of TIMP-3 in endometrial cancer may be caused by the aberrant expression of miR-103. Therefore, we conclude that miR-103 directly targets TIMP-3 3'-UTRs.

The roles of TIMP-3 in cancer progression have been highlighted in studies that have reported that the adenoviralmediated overexpression of TIMP-3 reduced the invasion of HeLa and HT1080 cells (17) and the vector-mediated expression of TIMP-3 in cancer cells reduced metastasis (18). In breast cancer, the overexpression of miR-21 was found to promote cell invasion via the regulation of TIMP-3 (19). TIMP-3 also induced cells to undergo apoptosis (20). Moreover, TIMP-3 was found to inhibit tumor growth in lung cancer cells in vivo (21).

In the present study, the results of the MTT assay revealed that the growth of cells transfected with anti-miR-103 was inhibited compared with the untreated and negative control groups. This finding suggests that the upregulation of TIMP-3 reduces cell proliferation, which is in agreement with the study by Finan et al (21), while the impact of modulated TIMP-3 on endometrial cancer in vivo requires further study. Notably, the results of the Annexin V-FITC versus PE assay showed that anti-miR-103 had no effect on the apoptosis of the Ishikawa and HEC-1B cells. One possible reason for this is that the biological effects of TIMP-3 are further defined by the specific genetic and epigenetic background of each cell line. Metastasis is a significant cause of cancer-related mortality. Therefore, identifying the role of miR-103 in invasion and metastasis has direct clinical implications. The results of the transwell invasion experiment showed that the transfection of anti-miR-103 reduced the invasion of endometrial cancer cells.

In conclusion, the results of the present study suggest that the tumor suppressor TIMP-3 is negatively regulated at the post-transcriptional level by miR-103 via a specific target motif at the TIMP-3 3'-UTR. Furthermore, miR-103 induces invasion and proliferation in endometrial cancer cells. Additional mechanisms and targets of miR-103 besides TIMP-3 are likely to contribute to miR-103-induced tumor cell proliferation and invasion. Although the aim of this study was to gain a better understanding of the function of miR-103 in endometrial cancer, future in vivo studies are required to address the therapeutic potential of miR-103.

\section{Acknowledgements}

The study was funded by general project H200942, supported by the Department of Public Health of Jiangsu Province. 


\section{References}

1. Calin GA, Sevignani C, Dumitru CD, Hyslop T, Noch E, Yendamuri S, Shimizu M, Rattan S, Bullrich F, Negrini M and Croce CM: Human microRNA genes are frequently located at fragile sites and genomic regions involved in cancers. Proc Natl Acad Sci USA 101: 2999-3004, 2004.

2. Yanaihara N, Caplen N, Bowman E, Seike M, Kumamoto K, Yi M, Stephens RM, Okamoto A, Yokota J, Tanaka T, et al: Unique microRNA molecular profiles in lung cancer diagnosis and prognosis. Cancer Cell 9: 189-198, 2006.

3. Xi Y, Shalgi R, Fodstad O, Pilpel Y and Ju J: Differentially regulated micro-RNAs and actively translated messenger RNA transcripts by tumor suppressor p53 in colon cancer. Clin Cancer Res 12: 2014-2024, 2006.

4. Wickramasinghe NS, Manavalan TT, Dougherty SM, Riggs KA, Li Y and Klinge CM: Estradiol downregulates miR-21 expression and increases miR-21 target gene expression in MCF-7 breast cancer cells. Nucleic Acids Res 37: 2584-2595, 2009.

5. Volinia S, Calin GA, Liu CG, Ambs S, Cimmino A, Petrocca F, Visone R, Iorio M, Roldo C, Ferracin M, et al: A microRNA expression signature of human solid tumors defines cancer gene targets. Proc Natl Acad Sci USA 103: 2257-2261, 2006.

6. Boren T, Xiong Y, Hakam A, Wenham R, Apte S, Wei Z, Kamath S, Chen DT, Dressman H and Lancaster JM: MicroRNAs and their target messenger RNAs associated with endometrial carcinogenesis. Gynecol Oncol 110: 206-215, 2008.

7. Chung TK, Cheung TH, Huen NY, Wong KW, Lo KW, Yim SF, Siu NS, Wong YM, Tsang PT, Pang MW, et al: Dysregulated microRNAs and their predicted targets associated with endometrioid endometrial adenocarcinoma in Hong Kong women. Int J Cancer 124: 1358-1365, 2009.

8. Gottardo F, Liu CG, Ferracin M, Calin GA, Fassan M, Bassi P, Sevignani C, Byrne D, Negrini M, Pagano F, et al: Micro-RNA profiling in kidney and bladder cancers. Urol Oncol 25: 387-392, 2007.

9. Yang GH, Wang F, Yu J, Wang XS, Yuan JY and Zhang JW: MicroRNAs are involved in erythroid differentiation control. J Cell Biochem 107: 548-556, 2009.

10. Guo Y, Chen Z, Zhang L, Zhou F, Shi S, Feng X, Li B, Meng X, Ma X, Luo M, et al: Distinctive microRNA profiles relating to patient survival in esophageal squamous cell carcinoma. Cancer Res 68: 26-33, 2008.

11. Moncini S, Salvi A, Zuccotti P, Viero G, Quattrone A, Barlati S, De Petro G, Venturin M and Riva P: The role of miR-103 and miR-107 in regulation of CDK5R1 expression and in cellular migration. PLoS One 6: e20038, 2011.
12. Feng $\mathrm{H}$, Cheung $\mathrm{AN}$ and Xue WC: Down-regulation and promoter methylation of tissue inhibitor of metalloproteinase 3 in choriocarcinoma. Gynecol Oncol 94: 375-382, 2004.

13. Yegnasubramanian S, Kowalski J, Gonzalgo ML, Zahurak M, Piantadosi S, Walsh PC, Bova GS, De Marzo AM, Isaacs WB and Nelson WG: Hypermethylation of $\mathrm{CpG}$ islands in primary and metastatic human prostate cancer. Cancer Res 64: 1975-1986, 2004.

14. Darnton SJ, Hardie LJ and Muc RS: Tissue inhibitor of metalloproteinase-3 (TIMP-3) gene is methylated in the development of esophageal adenocarcinoma: loss of expression correlates with poor prognosis. Int J Cancer 115: 351-358, 2005.

15. Bachman KE, Herman JG, Corn PG, Merlo A, Costello JF, Cavenee WK, Baylin SB and Graff JR: Methylation-associated silencing of the tissue inhibitor of metalloproteinase-3 gene suggest a suppressor role in kidney, brain, and other human cancers. Cancer Res 59: 798-802, 1999.

16. Kumar MS, Lu J, Mercer KL, Golub TR and Jacks T: Impaired microRNA processing enhances cellular transformation and tumorigenesis. Nat Genet 39: 673-677, 2007.

17. Baker AH, George SJ, Zaltsman AB, Murphy G and Newby AC: Inhibition of invasion and induction of apoptotic cell death of cancer cell lines by overexpression of TIMP-3. Br J Cancer 79: 1347-1355, 1999.

18. Lou Y, Yang X, Wang F, Cui Z and Huang Y: MicroRNA-21 promotes the cell proliferation, invasion and migration abilities in ovarian epithelial carcinomas through inhibiting the expression of PTEN protein. Int J Mol Med 26: 819-27, 2010.

19. Song B, Wang C, Liu J, Wang X, Lv L, Wei L, Xie L, Zheng Y and Song X: MicroRNA-21 regulates breast cancer invasion partly by targeting tissue inhibitor of metalloproteinase 3 expression. J Exp Clin Cancer Res 29: 29, 2010.

20. Bian J, Wang Y, Smith MR, Kim H, Jacobs C, Jackman J, Kung HF, Colburn NH and Sun Y: Suppression of in vivo tumor growth and induction of suspension cell death by tissue inhibitor of metalloproteinases (TIMP)-3. Carcinogenesis 17: 1805-1811, 1996.

21. Finan KM, Hodge G, Reynolds AM, Hodge S, Holmes MD, Baker AH and Reynolds PN: In vitro susceptibility to the proapoptotic effects of TIMP-3 gene delivery translates to greater in vivo efficacy versus gene delivery for TIMPs-1 or -2 . Lung Cancer 53: 273-284, 2006. 